\title{
Analysis and Design of Network-Based Control Systems with Binary Modulation
}

\author{
Shunli Zhao, Xunhe Yin, and Xueye Wei \\ School of Electronic and Information Engineering, Beijing Jiaotong University, Beijing 100044, China \\ Correspondence should be addressed to Xunhe Yin; xhyin@bjtu.edu.cn
}

Received 27 December 2013; Accepted 31 January 2014; Published 23 March 2014

Academic Editor: Linying Xiang

Copyright ( 2014 Shunli Zhao et al. This is an open access article distributed under the Creative Commons Attribution License, which permits unrestricted use, distribution, and reproduction in any medium, provided the original work is properly cited.

\begin{abstract}
Network-based control systems have been emerging technologies in control and computer communication fields over the past decade. This paper focuses on the analysis and design of network-based control systems with binary modulation. First, it is shown that different modulations can result in different delays which are inevitable in network-based control systems. The delay can be seen as constant delay when the transmission time is the main consideration. Second, channel noise can result in bit error while bit error is seen as active packet loss in this paper, in this context, the conditions of signal-to-noise ratio (SNR) for binary modulation that can guarantee the stability of systems are obtained according to the proposed algorithm. Third, the system with delay and noisy communication can be modeled as an asynchronous dynamic system (ADS); in addition, the stability is analyzed and controller is designed in terms of Lyapunov function and linear matrix inequality (LMI) scheme. Finally, without loss of generality, numerical simulation demonstrates the effectiveness of the proposed scheme and designed controller based on binary amplitude shift keying (2ASK) modulation.
\end{abstract}

\section{Introduction}

Network-based control systems, closing their feedback loops via networks or other shared communication mediums, have attracted much attention among researchers and engineers in the past decade. Critical issues that have received significant attention due to the addition of networks can be subjected to three main types of communication constraints: (1) limitations on bit rate; (2) medium access constraints; (3) transmission delay [1].

The study of modulation can be categorized into two directions, that is, quantization perspective and transmission perspective, from the existing literatures.

The quantization perspective belongs to the field of analog signals transmitted over a digital channel, in other words, digital transmission systems. In detail, analog signals need to be sampled, quantized, and coded before transmission in these transmission systems. In this respect, design of quantizer, uniform or nonuniform quantizer has been widely studied in network-based control systems [2].

Delta modulation, which is the simplest form of differential coding, has been investigated by many researchers to date. It is worth noting that the reason why this scheme is not called delta quantization or delta coding but delta modulation is that it is investigated from modulation perspective at the beginning, and this term is passed down in the following research. The other two similar terms are pulse code modulation (PCM) and differential PCM (DPCM). Substantially, the two are also coding methods. Hence, delta modulation can be seen as the special form of quantizer or coder because this modulation scheme not only has the function of quantization, but the function of coding. The output of delta modulation is a sequence of digital pulse signals produced by a two-level quantizer. It quantizes the difference between the current and the previous analog waveform rather than quantizing the absolute value of the analog waveform. Hence, the data capacity is decreased in comparison to that of quantizing the absolute value of the original signals.

Many researchers concentrate on delta modulation. The capability of delta modulation is studied in the context of network-based control systems in [3]. In this paper, a new modified scheme of the original form of delta modulation is proposed to improve the system stability. It is worth considering that this approach explicitly uses the system information 
to enhance the attraction domain. This result is extended to adaptive delta modulation (i.e., variable gain modulation) and multivariable linear systems in [4-6]. Recently, event triggering adaptive delta modulation is proposed to decrease the network traffic in a network-based control system that combines event triggered sampling with speech coding [7]. In engineering fields, Delta-Sigma modulation is used to compress and transmit the data from sensors and controllers based on a field programmable gate array (FPGA) experimental platform in [8].

The transmission perspective belongs to digital bandpass transmission in which coded signals are modulated to transmit over an analog channel. Note that this process is a way of signal transmission compared to delta modulation which is a way of quantization or coding. Different from delta modulation which is used for data compressing, this modulation scheme is applied to transmit signals according to the transmission requirements and environments. In this field, not many scholars pay their attention on network-based control systems with modulation. Binary phase shift keying (BPSK) modulation scheme is used to test the performance of the control system in the context of wireless sensor networks [9]. An adaptive modulation in terms of quadrature phase shift keying (QPSK) and quadrature amplitude modulation (QAM) is proposed in [10]. This adaptive modulation is formulated as an optimization problem, and the control command is derived by solving the optimization problem. However, the analysis and design of network-based control systems with modulation are not explicitly shown.

Time delay is an important characteristic of networkbased control systems. It significantly degrades the performance of control systems. Time delay possesses the stochastic property due to network congestion, routing, and noise. However, time delay can be modeled by constant delay in networks with binary modulation in this paper. That is to say, effects of congestion and routing can be ignored in this network context. So, many existing deterministic control strategies can be widely used to compensate for the effect of time delay.

The impact of bit error rate on network-based control systems has not been studied until now, while it is inevitable due to noise in signal transmission. The error codes are discarded if no error correct code is applied. This scheme is similar to that of active packet dropout method in congestion control. It is common in real-time control systems as either retransmission mechanism or error correct scheme will consume much time, which is harmful for the real-time performance of network-based control systems. In this respect, the error codes can be seen as the packets that are dropped actively, and, correspondingly, active packet loss rate can be replaced by bit error rate. Note that the packet loss is usually modeled either by a mutually independent stochastic process or by a Markov chain from the existing literatures in networkbased control systems. Nevertheless, the former neglects the interaction between the current dropped packet and the previous one, and it is impractical for the existing networks. The latter explicitly characterizes the real packet dropout in networks. Note that a significant element of Markov chain, transition probability matrix, is supposed to be known in advance in most works. However, it is difficult to be obtained in practical system design. This situation is changed if packet loss rate is replaced by bit error rate, and the rationality will be proved in the following sections. Meanwhile, the conditions that signal-to-noise ratio (SNR) satisfies are given to maintain the system stability. It is worth considering that SNR is an important performance index in signal transmission, and it is often ignored by many control system engineers.

In this context, the network-based control systems with time delay and channel noise can be modeled as an asynchronous dynamic system (ADS) based on bit error rate (i.e., active packet loss rate) in system design. Different from conventional ADS design where switching rate (also known as packet loss rate) is hard to be attained in advance, however, packet loss rate can be determined according to the modulation schemes applied in this paper. Then, the stability of system is analyzed and a controller is designed based on Lyapunov function and linear matrix inequality (LMI) scheme to stabilize a network-based control system with binary modulation.

The remainder of this paper is organized as follows. A linear system model and the assumptions used in this paper are given in Section 2. The communication channel characteristics containing time delay and noise are described in Section 3. In Section 4, the proposed method that packet loss rate is replaced by bit error rate is presented; then the stability is analyzed and controller is designed using Lyapunov function and LMI scheme based on ADS model. In Section 5, the effectiveness of the proposed algorithm and designed controller is demonstrated through a numerical illustration based on binary amplitude shift keying (2ASK) modulation. Finally, Section 6 gives conclusion and discussion.

Remark 1. Note that channel noise is different from the noise of control systems even though they all possess the random characteristic. The former does not affect the control systems as it is filtered in the demodulator side while the latter is the inherent noise that cannot be filtered in advance in control system design.

\section{System Dynamics}

Consider a linear time-invariant dynamical system whose dynamics can be described in the following:

$$
\begin{aligned}
\dot{\mathbf{x}}(t) & =\mathbf{A} \mathbf{x}(t)+\mathbf{B} \mathbf{u}(t), \\
\mathbf{y}(t) & =\mathbf{C} \mathbf{x}(t),
\end{aligned}
$$

where $\mathbf{x}(t) \in \mathbb{R}^{n}$ is the $N$-dimensional system state, $\mathbf{u}(t) \epsilon$ $\mathbb{R}^{m}$ is the $M$-dimensional control input, $\mathbf{y}(t) \in \mathbb{R}^{r}$ is the output, and $\mathbf{A}, \mathbf{B}$, and $\mathbf{C}$ are system gain matrices of appropriate dimension. It is worth noting that $\mathbf{A}, \mathbf{B}$, and $\mathbf{C}$ are definitely known as the system model is precisely constructed.

Diagram of a network-based control system is illustrated in Figure 1. $\mathbf{x}(k)$ and $\mathbf{u}(k)$ are the discrete forms of state $\mathbf{x}(t)$ and control input $\mathbf{u}(t)$, respectively. In this paper, networks only exist between sensors and controller. The measurements are modulated in the sensor side and demodulated in 


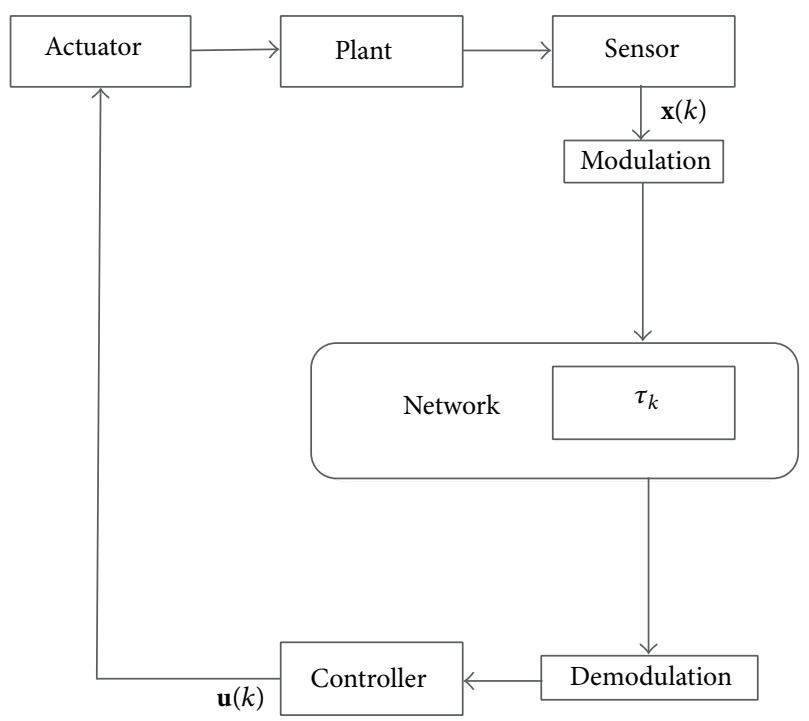

FIGURE 1: Diagram of a network-based control system.

the controller side. The control action $\mathbf{u}(k)$ computed by the controller is converted to electrical/electronic signal which is used by actuators to drive controlled plant.

To facilitate system analysis and design, the following assumptions about the network-based control systems are made.

Assumption 2. Sensors are time-driven, while controller and actuators are event-driven. This assumption avoids latency compared to time-driven controller and actuators.

Assumption 3. Assume that the delay is smaller than sampling period $T$. Hence, it can be denoted as $\tau_{k}<T$ at the $k$ th sampling instant. It is remarkable that $\tau_{k}$ only contains transmission delay (the amount of time required to push all of the packet's bits into the channel); that is, processing delay is negligible because of high speed routers used, propagation delay is ignored due to short distance communication, and queuing delay is neglected as few routers are deployed in networks. In addition, calculating delay of the controller is omitted here. We assume that there is no packet loss because of the reliable transmission. This is reasonable for a local area network with small scale.

Remark 4. No packet loss is assumed here, it means that the packet loss in propagation does not exist due to reliable communication. However, the packet with bit error is actively discarded when the packet arrives at the demodulator in the paper. Hence, the packet loss considered in this paper is different from the usual packet loss (i.e., passive packet loss) which results from heavy traffic load or unreliable transmission in network-based control systems.

\section{Communication Channel Characteristics}

In this section, time delay for a network-based control system with different modulations is discussed at first. Then, an important factor of noisy channel, bit error rate, is investigated based on different binary modulation schemes.
3.1. Time Delay. Suppose that there are $B$ bits that are used to be transmitted in each sampling period. Meanwhile, the transmission delay is the most important concern in network-based control systems while $B$ is sufficiently large. It is assumed that the symbol rate (or baud rate) is $R$ symbols per second, while each symbol contains $b$ bits data information (e.g., a BPSK symbol contains 1 bit data information). Hence, transmission delay at sampling instant $k$ can be computed:

$$
\tau_{k}=\frac{B}{b \cdot R}(s) .
$$

Remark 5. In this context, $\tau_{k}$ is inversely proportional to $b$ as the total bits $B$ and the symbol rate $R$ are fixed in each sampling period. So, $\tau_{k}$ is definite about each $b$. Note that there is no source coding or channel coding schemes used in this paper. Cyclic redundancy check (CRC) is only used to detect the bit error and no error correction code is used due to real-time requirements. There are no retransmission schemes used in information transmission due to the same reason above for a control system.

3.2. Noisy Channel. In communication systems, sum of useful signal and noise is received in the receiver. So, signal received possesses the random characteristic because of noise. Bit error is used to characterize the noise as noise in the channel can result in bit error in transmission systems.

In bandpass transmission, binary communication systems contain three typical types of modulation, that is, 2ASK, binary frequency shift keying (2FSK), and binary phase shift keying (2PSK). Bit error rate of each modulation with coherent demodulation is listed as below [11].

Bit error rate of $2 \mathrm{ASK}$ :

$$
P_{e a}=\frac{1}{2} \operatorname{erfc} \sqrt{\frac{r}{4}} .
$$

Similarly, bit error rate of 2FSK can be denoted as

$$
P_{e f}=\frac{1}{2} \operatorname{erfc} \sqrt{\frac{r}{2}} .
$$

Bit error rate of 2PSK is

$$
P_{e p}=\frac{1}{2} \operatorname{erfc} \sqrt{r},
$$

where $r$ denotes the SNR for the specific modulation and the complementary error function $\operatorname{erfc}(x)=1-(2 / \sqrt{\pi})$ $\int_{0}^{x} e^{-z^{2}} \mathrm{~d} z$.

\section{Stability Analysis and Controller Design}

In this section, the network-based control system with time delay and bit error is modeled as an ADS at first. Then, the stable SNR theorem is given according to the proposed method. Finally, stability analysis is made and controller is designed based on Lyapunov function and LMI scheme.

4.1. ADS System Model and Control Law. The networkbased control systems with packet dropout can be modeled 
as an ADS. The measurements are said to be transmitted successfully if the measurements are received and used by controller, this event is denoted by S1. Correspondingly, if the measurements are discarded because of bit error, then it is denoted by S2.

The data that controller receives can be denoted as (6) due to time delay when S1 occurs:

$$
\widetilde{\mathbf{x}}(k)= \begin{cases}\widetilde{\mathbf{x}}(k-1), & K T<t \leq K T+\tau, \\ \mathbf{x}(k), & K T+\tau<t \leq(K+1) T .\end{cases}
$$

Then, the data is discarded when S2 occurs; however, in this context, controller can use the data stored in zero-order hold $(\mathrm{ZOH})$. So, the data that controller used can be denoted as

$$
\widetilde{\mathbf{x}}(k)=\widetilde{\mathbf{x}}(k-1) .
$$

Discretizing (1) with sampling period $T$, the networkbased control systems with time delay and channel noise can be written as

$$
\begin{aligned}
& S 1:\left\{\begin{array}{l}
\mathbf{x}(k+1)=\mathbf{A}_{s} \mathbf{x}(k)+\mathbf{B}_{s 1}(\tau) \mathbf{u}(k)+\mathbf{B}_{s 2}(\tau) \mathbf{u}(k-1) \\
\widetilde{\mathbf{x}}(k)=\mathbf{x}(k),
\end{array}\right. \\
& S 2:\left\{\begin{array}{l}
\mathbf{x}(k+1)=\mathbf{A}_{s} \mathbf{x}(k)+\mathbf{B}_{s}(\tau) \mathbf{u}(k-1) \\
\widetilde{\mathbf{x}}(k)=\widetilde{\mathbf{x}}(k-1),
\end{array}\right.
\end{aligned}
$$

where $\mathbf{A}_{s}=e^{\mathbf{A} T}, \mathbf{B}_{s 1}(\tau)=\int_{0}^{T-\tau} e^{\mathbf{A} t} \mathrm{~d} t \cdot \mathbf{B}, \mathbf{B}_{s 2}(\tau)=\int_{T-\tau}^{T} e^{\mathbf{A} t} \mathrm{~d} t$. $\mathbf{B}$, and $\mathbf{B}_{s}(\tau)=\int_{0}^{T} e^{\mathbf{A} t} \mathrm{~d} t \cdot \mathbf{B}$.

The discrete state feedback controller used in this network-based control system can be written as

$$
\mathbf{u}(k)=\mathbf{K} \widetilde{\mathbf{x}}(k) .
$$

Then, substituting (9) into (8) and letting $\mathbf{z}(k)=\left(\mathbf{x}^{T}(k)\right.$, $\left.\widetilde{\mathbf{x}}^{T}(k-1)\right)^{T}$, the closed-loop system can be obtained:

$$
\begin{aligned}
& \text { S1: }\left[\begin{array}{c}
\mathbf{x}(k+1) \\
\widetilde{\mathbf{x}}(k)
\end{array}\right]=\left[\begin{array}{cc}
\mathbf{A}_{s}+\mathbf{B}_{s 1}(\tau) \mathbf{K} & \mathbf{B}_{s 2}(\tau) \mathbf{K} \\
\mathbf{I} & \mathbf{0}
\end{array}\right]\left[\begin{array}{c}
\mathbf{x}(k) \\
\widetilde{\mathbf{x}}(k-1)
\end{array}\right], \\
& S 2:\left[\begin{array}{c}
\mathbf{x}(k+1) \\
\widetilde{\mathbf{x}}(k)
\end{array}\right]=\left[\begin{array}{cc}
\mathbf{A}_{s} & \mathbf{B}_{s}(\tau) \mathbf{K} \\
\mathbf{I} & \mathbf{0}
\end{array}\right]\left[\begin{array}{c}
\mathbf{x}(k) \\
\widetilde{\mathbf{x}}(k-1)
\end{array}\right] .
\end{aligned}
$$

4.2. Stable SNR Theorem. In this paper, packet loss rate is replaced by bit error rate. This scheme is reasonable as the error bit is evenly distributed. Each packet only has one error bit, while the case of two error bits for each packet can be ignored due to the lower probability of occurrence. For example, assume that bit error rate $P_{e}=10^{-5}$, and the probability of one error bit in a packet containing 8 bits is $P_{1}=C_{8}^{1} \times 10^{-5}=8 \times 10^{-5}$; however, the probability of two error bits in a packet is $P_{2}=C_{8}^{2} \times P_{e}^{2}=2.8 \times 10^{-10}$. Hence, the case of two error bits can be neglected due to $P_{2} \ll P_{1}$.
Theorem 6 (see [12]). Suppose that the closed-loop system with no packet loss is stable.

(1) If the open-loop system is marginally stable, then the system is exponentially stable for all $0<\gamma \leq 1$.

(2) If the open-loop system is unstable, then the system is exponentially stable for all $1 /\left(1-\gamma_{1} / \gamma_{2}\right)<\gamma \leq 1$, where $\gamma$ denotes the packet loss rate, $\gamma_{1}=\lg \left[\lambda_{\max }^{2}(\Phi-\Gamma \mathbf{K})\right]$, $\gamma_{2}=\lg \left[\lambda_{\max }^{2}(\boldsymbol{\Phi})\right], \boldsymbol{\Phi}=e^{\mathbf{A} T}, \boldsymbol{\Gamma}=\int_{0}^{T} e^{\mathbf{A} s} d s \cdot \mathbf{B}$, and $\lambda_{\max }(\boldsymbol{\Phi}-\boldsymbol{\Gamma} \mathbf{K})$ denotes the maximal eigenvalue of $(\boldsymbol{\Phi}-\boldsymbol{\Gamma} \mathbf{K})$ while $\lambda_{\max }(\boldsymbol{\Phi})$ the maximal eigenvalue of $\boldsymbol{\Phi}$. $\mathbf{K}$ denotes the feedback gain.

Therefore, the following theorem is easy to be obtained according to the proposed algorithm that packet loss rate is replaced by bit error rate.

Stable SNR Theorem. Suppose the closed-loop system with no packet loss is stable, and packet loss rate can be replaced by bit error rate. The bound of SNR that makes the system stable should satisfy the following conditions.

(1) If the open-loop system is marginally stable, then the system is exponentially stable for all $0<p_{e a} \leq 1$ for 2ASK, $0<p_{e f} \leq 1$ for $2 \mathrm{FSK}$, and $0<p_{e p} \leq 1$ for 2PSK.

(2) If the open-loop system is unstable, then the system is exponentially stable for all $1 /\left(1-\gamma_{1} / \gamma_{2}\right)<P_{e a} \leq$ 1 for 2 ASK, $1 /\left(1-\gamma_{1} / \gamma_{2}\right)<P_{e f} \leq 1$ for 2 FSK, and $1 /\left(1-\gamma_{1} / \gamma_{2}\right)<P_{e p} \leq 1$ for 2 PSK, where $\gamma_{1}, \gamma_{2}, \Phi$, and $\Gamma$ are the same as in Theorem 6 .

\subsection{Stability Analysis and Controller Design}

\subsubsection{Related Definitions and Lemmas}

Definition 7. An ADS is said to be exponential stable if there exists a constant $\alpha$ such that

$$
\lim _{k \rightarrow \infty} \alpha^{k}\left\|\mathbf{x}_{k}\right\|=0 \text {. }
$$

Lemma 8 (see [13]). For an ADS, if there exists a Lyapunov function $V: \mathbb{R}^{n} \rightarrow \mathbb{R}_{+}$and $V$ satisfies $\beta_{1}\|\mathbf{x}\|^{2} \leq V(\mathbf{x}) \leq$ $\beta_{2}\|\mathbf{x}\|^{2}$, where $\beta_{1}>0, \beta_{2}>0$, and there exist scalars $\alpha>0$, $\alpha_{i}>0, i=1 \cdots N$, such that $V\left(\mathbf{x}_{k+1}\right)-V\left(\mathbf{x}_{k}\right) \leq\left(\alpha_{s}^{-2}-1\right) V\left(\mathbf{x}_{k}\right)$ and $\alpha_{1}^{r_{1}} \alpha_{2}^{r_{2}} \cdots \alpha_{N}^{r_{N}}>\alpha>1$, where $0<r_{s} \leq 1, s=1 \cdots N$ denotes the occurrence rate for each subsystem, then this ADS is exponentially stable with decay rate $\alpha$.

Remark 9. Note that $r_{s}$ is the same as $\gamma$ in Theorem 6.

Schur Complement Lemma 10 (see [14]). Consider a symmetric matrix $X$ that can be partitioned into blocks:

$$
\mathbf{X}=\left[\begin{array}{cc}
\mathbf{A} & \mathbf{B} \\
\mathbf{B}^{T} & \mathbf{C}
\end{array}\right],
$$

where both $\mathbf{A}$ and $\mathbf{C}$ are symmetric, square, and negative definite; then the following properties are equivalent:

(1) $\mathbf{X}$ is negative semidefinite;

(2) the Schur complement of $\mathbf{C}$ in $\mathbf{X}$, denoted as $\mathbf{A}-\mathbf{B C B}^{T}$, is negative semidefinite; 
(3) the Schur complement of $\mathbf{A}$ in $\mathbf{X}$, denoted as $\mathbf{C}-\mathbf{B}^{T} \mathbf{A B}$, is negative semidefinite.

\subsubsection{Stability Analysis}

Theorem 11. Closed-loop network-based control system (10) with active packet loss rate $r$ is said to be asymptotically stable if there exist symmetric positive definite matrices $\mathbf{P}, \mathbf{Q}$ and feedback gain matrix $\mathbf{K}$ satisfying the following inequalities:

$$
\begin{aligned}
& \alpha_{1}^{r} \times \alpha_{2}^{1-r}>\alpha>1 \\
& {\left[\begin{array}{ccc}
-\mathbf{P}^{-1} & \mathbf{A}_{s}+\mathbf{B}_{s 1}(\tau) \mathbf{K} & \mathbf{B}_{s 2}(\tau) \mathbf{K} \\
\left(\mathbf{A}_{s}+\mathbf{B}_{s 1}(\tau) \mathbf{K}\right)^{T} & \mathbf{Q}-\alpha_{1}^{-2} \mathbf{P} & \mathbf{0} \\
\left(\mathbf{B}_{s 2}(\tau) \mathbf{K}\right)^{T} & \mathbf{0} & -\alpha_{1}^{-2} \mathbf{Q}
\end{array}\right]<0,} \\
& V(\mathbf{x}(k+1))-\alpha_{1}^{-2} V(\mathbf{x}(k)) \\
& =\mathbf{x}^{T}(k+1) \mathbf{P} \mathbf{x}(k+1)+\widetilde{\mathbf{x}}^{T}(k) \mathbf{Q} \widetilde{\mathbf{x}}(k)-\alpha_{1}^{-2} \mathbf{x}^{T}(k) \mathbf{P} \mathbf{x}(k)-\alpha_{1}^{-2} \widetilde{\mathbf{x}}^{T}(k-1) \mathbf{Q} \widetilde{\mathbf{x}}(k-1) \\
& =\left[\left(\mathbf{A}_{s}+\mathbf{B}_{s 1}(\tau) \mathbf{K}\right) \mathbf{x}(k)+\mathbf{B}_{s 2}(\tau) \mathbf{K} \widetilde{\mathbf{x}}(k-1)\right]^{T} \mathbf{P}\left[\left(\mathbf{A}_{s}+\mathbf{B}_{s 1}(\tau) \mathbf{K}\right) \mathbf{x}(k)+\mathbf{B}_{s 2}(\tau) \mathbf{K} \widetilde{\mathbf{x}}(k-1)\right] \\
& +\widetilde{\mathbf{x}}(k) \mathbf{Q} \widetilde{\mathbf{x}}(k)-\alpha_{1}^{-2} \mathbf{x}^{T}(k) \mathbf{P} \mathbf{x}(k)-\alpha_{1}^{-2} \widetilde{\mathbf{x}}^{T}(k-1) \mathbf{Q} \widetilde{\mathbf{x}}(k-1) \\
& =\mathbf{x}^{T}(k)\left[\left(\mathbf{A}_{s}+\mathbf{B}_{s 1}(\tau) \mathbf{K}\right)^{T} \mathbf{P}\left(\mathbf{A}_{s}+\mathbf{B}_{s 1}(\tau) \mathbf{K}\right)+\mathbf{Q}-\alpha_{1}^{-2} \mathbf{P}\right] \mathbf{x}(k) \\
& +\mathbf{x}^{T}(k)\left[\left(\mathbf{A}_{s}+\mathbf{B}_{s 1}(\tau) \mathbf{K}\right)^{T} \mathbf{P B}_{s 2}(\tau) \mathbf{K}\right] \widetilde{\mathbf{x}}(k-1) \\
& +\widetilde{\mathbf{x}}(k-1)\left[\left(\mathbf{B}_{s 2}(\tau) \mathbf{K}\right)^{T} \mathbf{P}\left(\mathbf{A}_{s}+\mathbf{B}_{s 1}(\tau) \mathbf{K}\right)\right] \mathbf{x}(k) \\
& +\widetilde{\mathbf{x}}^{T}(k-1)\left[\left(\mathbf{B}_{s 2}(\tau) \mathbf{K}\right)^{T} \mathbf{P}\left(\mathbf{B}_{s 2}(\tau) \mathbf{K}\right)-\alpha_{1}^{-2} \mathbf{Q}\right] \widetilde{\mathbf{x}}(k-1) \\
& =\mathbf{z}^{T}(k)\left[\begin{array}{cc}
\left(\mathbf{A}_{s}+\mathbf{B}_{s 1}(\tau) \mathbf{K}\right)^{T} \mathbf{P}\left(\mathbf{A}_{s}+\mathbf{B}_{s 1}(\tau) \mathbf{K}\right)+\mathbf{Q}-\alpha_{1}^{-2} \mathbf{P} & \left(\mathbf{A}_{s}+\mathbf{B}_{s 1}(\tau) \mathbf{K}\right)^{T} \mathbf{P} \mathbf{B}_{s 2}(\tau) \mathbf{K} \\
\left(\mathbf{B}_{s 2}(\tau) \mathbf{K}\right)^{T} \mathbf{P}\left(\mathbf{A}_{s}+\mathbf{B}_{s 1}(\tau) \mathbf{K}\right) & \left(\mathbf{B}_{s 2}(\tau) \mathbf{K}\right)^{T} \mathbf{P}\left(\mathbf{B}_{s 2}(\tau) \mathbf{K}\right)-\alpha_{1}^{-2} \mathbf{Q}
\end{array}\right] \mathbf{z}(k) .
\end{aligned}
$$

By Schur complement lemma, then

$$
\begin{aligned}
V(\mathbf{x}(k+1))-\alpha_{1}^{-2} V(\mathbf{x}(k)) \\
\quad=\left[\begin{array}{ccc}
-\mathbf{P}^{-1} & \mathbf{A}_{s}+\mathbf{B}_{s 1}(\tau) \mathbf{K} & \mathbf{B}_{s 2}(\tau) \mathbf{K} \\
\left(\mathbf{A}_{s}+\mathbf{B}_{s 1}(\tau) \mathbf{K}\right)^{T} & \mathbf{Q}-\alpha_{1}^{-2} \mathbf{P} & \mathbf{0} \\
\left(\mathbf{B}_{s 2}(\tau) \mathbf{K}\right)^{T} & \mathbf{0} & -\alpha_{1}^{-2} \mathbf{Q}
\end{array}\right] .
\end{aligned}
$$

According to Lemma 8, (13b) holds.

Case 2. Consider

$$
\begin{aligned}
V(\mathbf{x} & (k+1))-\alpha_{2}^{-2} V(\mathbf{x}(k)) \\
= & \mathbf{x}^{T}(k+1) \mathbf{P} \mathbf{x}(k+1)+\widetilde{\mathbf{x}}^{T}(k) \mathbf{Q} \widetilde{\mathbf{x}}(k)-\alpha_{2}^{-2} \mathbf{x}^{T}(k) \mathbf{P} \mathbf{x}(k)-\alpha_{2}^{-2} \widetilde{\mathbf{x}}^{T}(k-1) \mathbf{Q} \widetilde{\mathbf{x}}(k-1) \\
= & {\left[\mathbf{A}_{s} \mathbf{x}(k)+\mathbf{B}_{s}(\tau) \mathbf{K} \widetilde{\mathbf{x}}(k-1)\right]^{T} \mathbf{P}\left[\mathbf{A}_{s} \mathbf{x}(k)+\mathbf{B}_{s}(\tau) \mathbf{K} \widetilde{\mathbf{x}}(k-1)\right]+\widetilde{\mathbf{x}}^{T}(k) \mathbf{Q} \widetilde{\mathbf{x}}(k)-\alpha_{2}^{-2} \mathbf{x}^{T}(k) \mathbf{P} \mathbf{x}(k) } \\
& -\alpha_{2}^{-2} \widetilde{\mathbf{x}}(k-1) \mathbf{Q} \widetilde{\mathbf{x}}(k-1) \\
= & \mathbf{x}^{T}(k)\left[\mathbf{A}_{s}^{T} \mathbf{P} \mathbf{A}_{s}-\alpha_{2}^{-2} \mathbf{P}\right] \mathbf{x}(k)+\mathbf{x}^{T}(k)\left[\mathbf{A}_{s}^{T} \mathbf{P}\left(\mathbf{B}_{s}(\tau) \mathbf{K}\right)\right] \widetilde{\mathbf{x}}(k-1)+\mathbf{x}^{T}(k-1)\left[\left(\mathbf{B}_{s}(\tau) \mathbf{K}\right)^{T} \mathbf{P} \mathbf{A}_{s}\right] \mathbf{x}(k) \\
& +\widetilde{\mathbf{x}}^{T}(\mathbf{k}-\mathbf{1})\left[\left(\mathbf{B}_{s}(\tau) \mathbf{K}\right)^{T} \mathbf{P}\left(\mathbf{B}_{s}(\tau) \mathbf{K}\right)-\left(1-\alpha_{2}^{-2}\right) \mathbf{Q}\right] \widetilde{\mathbf{x}}(k-1) \\
= & \mathbf{z}^{T}(k)\left[\begin{array}{l}
\mathbf{A}_{s}^{T} \mathbf{P} \mathbf{A}_{s}-\alpha_{2}^{-2} \mathbf{P} \\
\left(\mathbf{B}_{s}(\tau) \mathbf{K}\right)^{T} \mathbf{P} \mathbf{A}_{s}\left(\mathbf{B}_{s}(\tau) \mathbf{K}\right)^{T} \mathbf{P}\left(\mathbf{B}_{s}^{T} \mathbf{P}\left(\mathbf{B}_{s}(\tau) \mathbf{K}\right)-\left(1-\alpha_{2}^{-2}\right) \mathbf{Q}\right]
\end{array}\right] \mathbf{z}(k) .
\end{aligned}
$$


By Schur complement lemma, then

$$
\begin{aligned}
V(\mathbf{x}(k+1))-\alpha_{2}^{-2} V(\mathbf{x}(k)) \\
\quad=\left[\begin{array}{ccc}
-\mathbf{P}^{-1} & \mathbf{A}_{s} & \mathbf{B}_{s}(\tau) \mathbf{K} \\
\mathbf{A}_{s}^{T} & -\alpha_{2}^{-2} \mathbf{P} & \mathbf{0} \\
\left(\mathbf{B}_{s}(\tau) \mathbf{K}\right)^{T} & \mathbf{0} & \left(1-\alpha_{2}^{-2}\right) \mathbf{Q}
\end{array}\right] .
\end{aligned}
$$

According to Lemma 8, (13c) holds.

Hence, the network-based control system (10) is asymptotically stable.

4.3.3. Controller Parameter Design. Note that inequalities (13b) and (13c) are not LMIs. In order to obtain feasible LMIs, (13b) and (13c) are premultiplied and postmultiplied

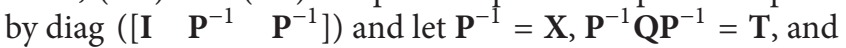
$\mathbf{K X}=\mathbf{Y}$. We can obtain

$$
\begin{gathered}
\alpha_{1}^{r} \times \alpha_{2}^{1-r}>\alpha>1, \\
{\left[\begin{array}{ccc}
-\mathbf{X} & \mathbf{A}_{s} \mathbf{X}+\mathbf{B}_{s 1}(\tau) \mathbf{Y} & \mathbf{B}_{s 2}(\tau) \mathbf{Y} \\
* & \mathbf{T}-\alpha_{1}^{-2} \mathbf{X} & \mathbf{0} \\
* & * & -\alpha_{1}^{-2} \mathbf{T}
\end{array}\right]<0,} \\
{\left[\begin{array}{ccc}
-\mathbf{X} & \mathbf{A}_{s} \mathbf{X} & \mathbf{B}_{s}(\tau) \mathbf{Y} \\
* & -\alpha_{2}^{-2} \mathbf{X} & \mathbf{0} \\
* & * & \left(1-\alpha_{2}^{-2}\right) \mathbf{T}
\end{array}\right]<0,}
\end{gathered}
$$

where "*" denotes the symmetric entries. $\mathbf{X}, \mathbf{T}$, and $\mathbf{Y}$ can be solved by LMI toolbox. Obviously, $\mathbf{Y}=\mathbf{K} \cdot \mathbf{X}^{-1}$ as $\mathbf{X}$ is a symmetric and positive definite matrix.

\section{Numerical Simulation}

In this section, a numerical simulation is given to demonstrate the effectiveness of the proposed algorithm and the designed control law. Without loss of generality, 2ASK scheme is investigated here. Similar analysis can be made on 2PSK and 2FSK.

In this control system, network only exists between sensors and controller, information collected by sensors is transmitted to controller after modulating. When the information arrives, controller computes the control commands to manipulate the system output.

The dynamics of controlled plant is given as follows:

$$
\mathbf{A}=\left[\begin{array}{cc}
0 & 1 \\
-1 & -2
\end{array}\right], \quad \mathbf{B}=\left[\begin{array}{l}
0 \\
1
\end{array}\right]
$$

It is assumed that the measured SNR is $11.2 \mathrm{~dB}$ here. Hence, bit error rate for $2 \mathrm{ASK}$ is 0.01 according to (3). Suppose that the sampling period $T$ is $10 \mathrm{~ms}$, and the baud rate is $20 \mathrm{~K}$ baud, that is, $20 \mathrm{~K}$ symbols per second. Assume that the length of frame is 100 bit, in this context, delay of $2 \mathrm{ASK}$ is $5 \mathrm{~ms}$ according to (2). The initial state is $(1.5,-1)$. We choose $\alpha_{1}=1.07$ and $\alpha_{2}=0.76$; then $\alpha=1.3422$.

Hence, control law of system with 2 ASK modulation is

$$
\mathbf{K}_{A}=\left[\begin{array}{ll}
-0.0611 & -2.1878
\end{array}\right] \text {. }
$$

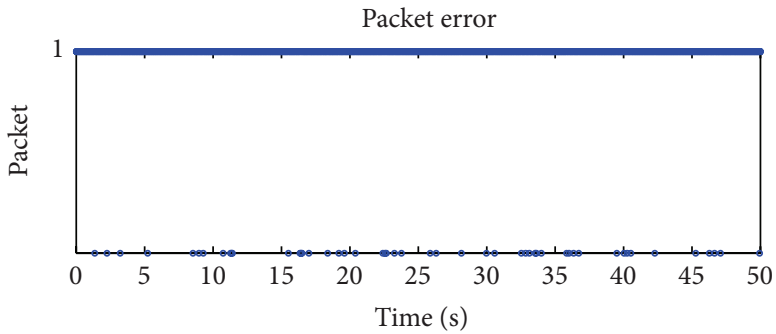

FIGURE 2: Packet error illustration.

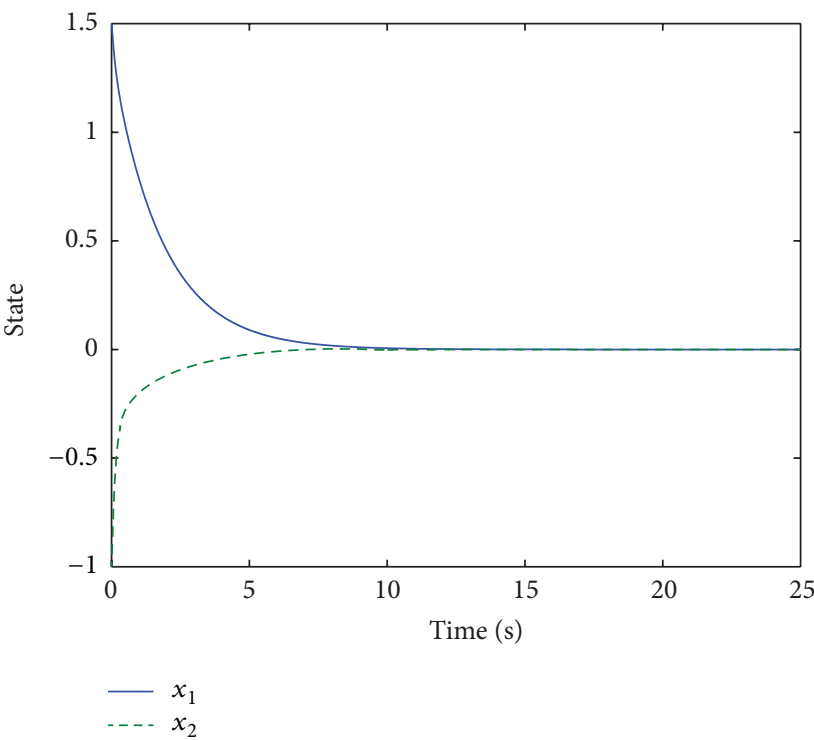

FIGURE 3: State response illustration with 2ASK modulation.

Packet error illustration with 2ASK modulation is shown in Figure 2.

In Figure 2, Packet $=0$ means that the packet is dropped due to bit error while Packet $=1$ denotes the packet without bit error. This diagram only denotes the packet error or not, so the values between 0 and 1 are meaningless and will never be used. Controller will use the data stored in $\mathrm{ZOH}$ when the packet is dropped. State response illustration with 2ASK modulation is shown in Figure 3.

The system states, $x_{1}$ and $x_{2}$, converge to the equilibrium from Figure 3. That is, this network-based control system with 2ASK modulation and noisy channel is stable. Hence, the proposed algorithm is reasonable and the designed controller is effective in this system design.

\section{Conclusion and Discussion}

The analysis and design of network-based control systems with binary modulation are studied in this paper. It is shown that different modulations can result in different delays which are important for network-based control systems. Delay in networks with modulation can be modeled as constant delay when transmission delay is the main consideration. Furthermore, a novel active packet loss rate method is proposed in noisy communication. Compared to the existing scheme of 
packet loss rate which is hard to be determined in application, the proposed scheme can obtain the packet loss rate based on the measured SNR for the specific modulation scheme. Meantime, the stable condition for each binary modulation scheme is given. Finally, without loss of generality, 2ASK modulation is chosen to demonstrate the effectiveness of the proposed algorithm and the designed control law.

This paper focuses on simple but typical modulations in application, such as $2 \mathrm{ASK}, 2 \mathrm{FSK}$, and 2PSK. The same idea also can be used to M-ary modulation. In addition, passive packet loss is not considered in this paper, noisy communication combining passive packet loss with bit error rate may be considered in our future work. Note that the stable SNR theorem only gives the conditions of modulation ensuring the stability of the network-based control systems as the complementary error function has no analytic solution; the simpler form of the stable SNR theorem may be obtained in the future.

\section{Conflict of Interests}

The authors declare that there is no conflict of interests regarding the publication of this paper.

\section{Acknowledgment}

This work is supported by National Natural Science Foundation of China (NSFC) under Grant no. 61172022.

\section{References}

[1] D. Hristu-Varsakelis, "Stabilization of networked control systems with access constraints and delays," in Proceedings of the 45th IEEE Conference on Decision and Control (CDC '06), pp. 1123-1128, San Diego, Calif, USA, December 2006.

[2] L. Li and F. Y. Wang, "Control and communication synthesis in networked control systems," International Journal of Intelligent Control and Systems, vol. 13, no. 2, pp. 81-88, 2008.

[3] C. Canudas-de-Wit, F. R. Rubio, J. Fornés, and F. Gómez-Estern, "Differential coding in networked controlled linear systems," in Proceedings of the American Control Conference, pp. 4177-4182, Minneapolis, Minn, USA, June 2006.

[4] C. Canudas-de-Wit, F. Gómez-Estern, and F. R. Rubio, "Deltamodulation coding redesign for feedback-controlled systems," IEEE Transactions on Industrial Electronics, vol. 56, no. 7, pp. 2684-2696, 2009.

[5] F. Gómez-Estern, C. Canudas-De-Wit, and F. R. Rubio, "Adaptive delta modulation in networked controlled systems with bounded disturbances," IEEE Transactions on Automatic Control, vol. 56, no. 1, pp. 129-134, 2011.

[6] J. Jaglin, C. Canudas-De-Wit, and C. Siclet, "Delta modulation for multivariable centralized linear networked controlled systems," in Proceedings of the 47th IEEE Conference on Decision and Control (CDC '08), pp. 4910-4915, Cancun, Mexico, December 2008.

[7] U. Premaratne, S. K. Halgamuge, I. M. Y. Mareels et al., "Event triggered adaptive differential modulation: a new method for traffic reduction in networked control systems," IEEE Transactions on Automatic Control, vol. 58, no. 7, pp. 1696-1706, 2013.
[8] T. Li and Y. Fujimoto, "Control system with high-speed and realtime communication links," IEEE Transactions on Industrial Electronics, vol. 55, no. 4, pp. 1548-1557, 2008.

[9] M. Trivellato and N. Benvenuto, "State control in networked control systems under packet drops and limited transmission bandwidth," IEEE Transactions on Communications, vol. 58, no. 2, pp. 611-622, 2010.

[10] H. S. Li, J. B. Song, Q. Zeng et al., "Adaptive Modulation in Networked Control Systems with Application in Smart Grids," IEEE Communication Letters, vol. 17, no. 7, pp. 1305-1308, 2013.

[11] S. Haykin, Communications Systemsedition, Wiley, New York, NY, USA, 4th edition, 2001.

[12] J. Nilsson, Real-time Control Systems with Delays [Ph.D. dissertation], Department of Automatic Control, Lund Institute of Technology, Lund, Sweden, 1998.

[13] A. Rabello and A. Bhaya, "Stability of asynchronous dynamical systems with rate constraints and applications," IEE Proceedings on Control Theory and Applications, vol. 150, no. 5, pp. 546-550, 2003.

[14] S. Boyd and L. Vandenberghe, Convex Optimization, Cambridge University Press, Cambridge, UK, 2004. 


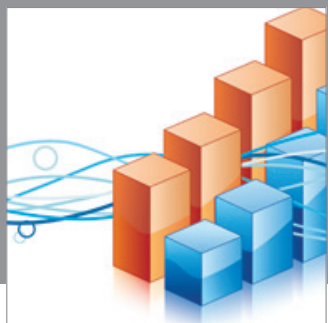

Advances in

Operations Research

mansans

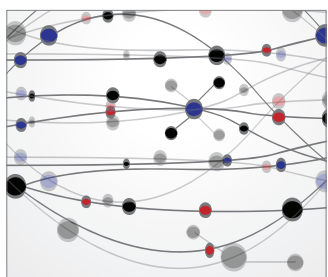

The Scientific World Journal
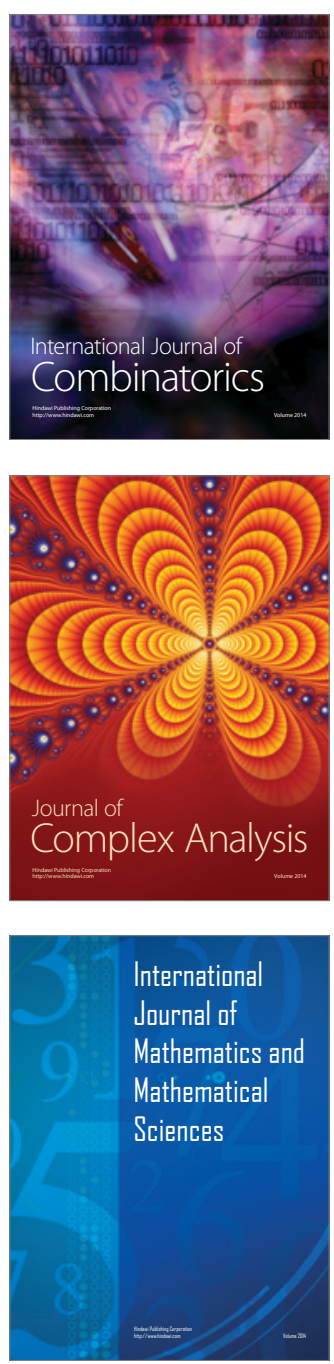
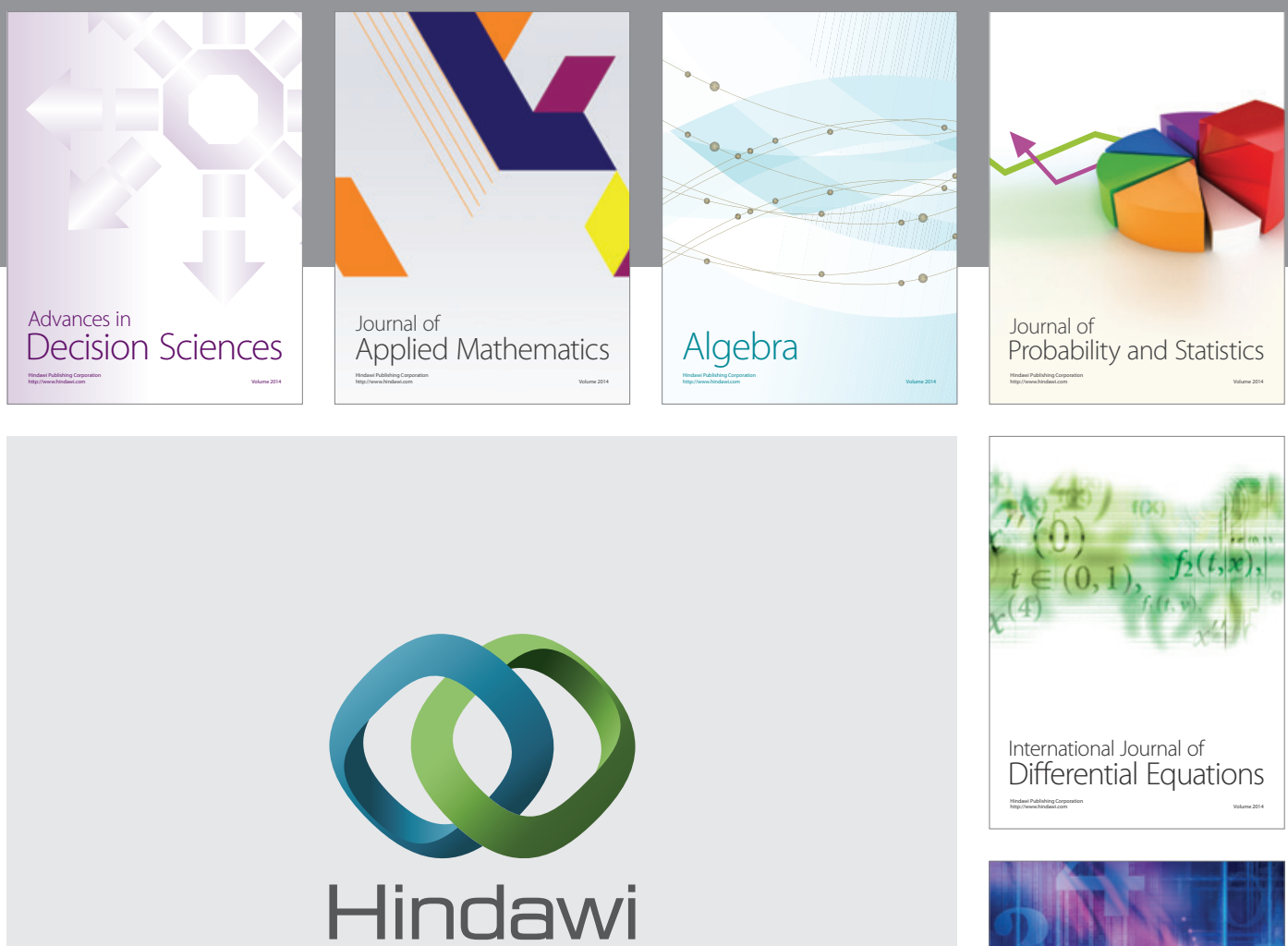

Submit your manuscripts at http://www.hindawi.com
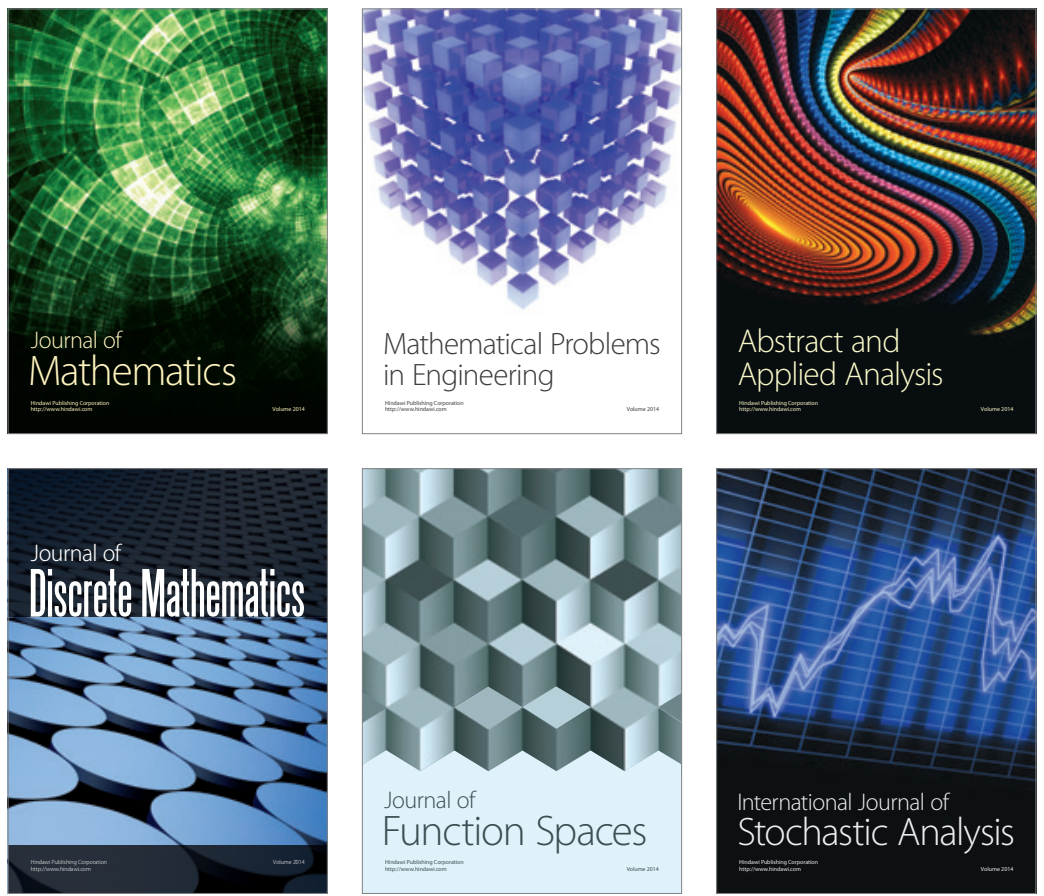

Journal of

Function Spaces

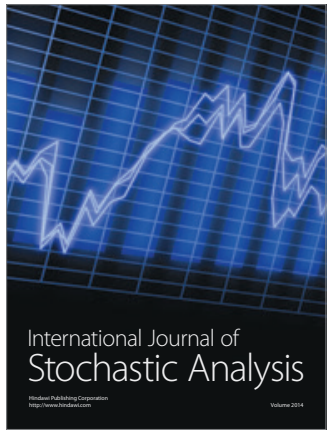

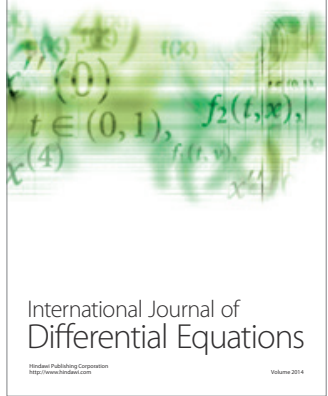
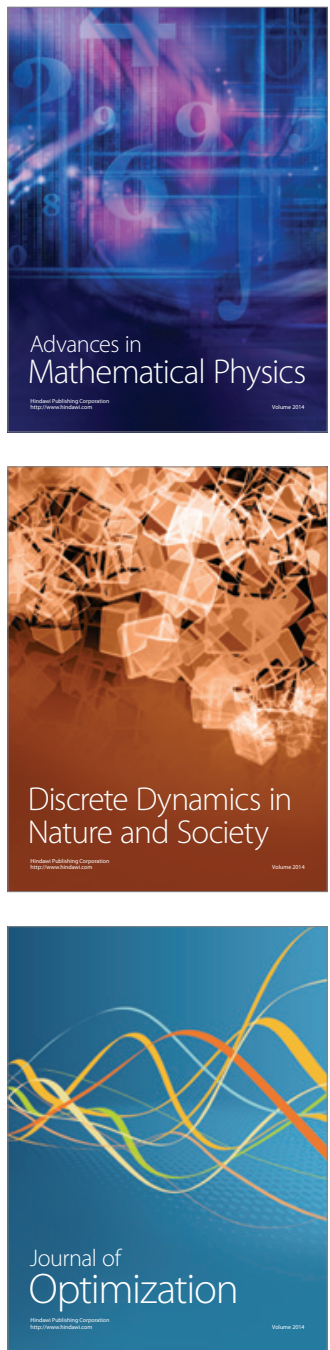